\title{
Cathelicidin antimicrobial protein, vitamin D, and risk of death in critically ill patients
}

\author{
David E Leaf*, Heather E Croy, Sara J Abrahams, Anas Raed and Sushrut S Waikar
}

\begin{abstract}
Introduction: Decreased production of cathelicidin antimicrobial protein-18 (hCAP18) has been proposed to be a key mechanism linking decreased 25-hydroxyvitamin D (25D) levels with adverse outcomes among critically ill patients. However, few studies in humans have directly assessed plasma hCAP18 levels, and no study has evaluated the association between hCAP18 levels and adverse outcomes among critically ill patients.

Methods: We performed a single-center, prospective cohort study among 121 critically ill patients admitted to intensive care units (ICUs) between 2008 and 2012. We measured plasma hCAP18, 25D, D-binding protein, and parathyroid hormone levels on ICU day 1. The primary endpoint was 90-day mortality. Secondary endpoints included hospital mortality, sepsis, acute kidney injury, duration of mechanical ventilation, and hospital length of stay.
\end{abstract}

Results: ICU day 1 hCAP18 levels were directly correlated with 25D levels (Spearman's rho $\left(r_{s}\right)=0.30, P=0.001$ ). In multivariate analyses adjusted for age and Acute Physiology and Chronic Health Evaluation II (APACHE II) score, patients with hCAP18 levels in the lowest compared to highest tertile on ICU day 1 had a 4.49 (1.08 to 18.67) greater odds of 90-day mortality, and also had greater odds of sepsis. ICU day 1 levels of other analytes were not associated with 90-day mortality.

Conclusions: Lower 25D levels on ICU day 1 are associated with lower hCAP18 levels, which are in turn associated with a greater risk of 90-day mortality. These findings provide a potential mechanistic basis for the frequently observed association between low 25D levels and poor outcomes in critically ill patients.

\section{Introduction}

Vitamin D deficiency/insufficiency is very common in critically ill patients, with reported rates ranging from $81.5 \%$ to $99 \%$ [1-3]. Further, multiple studies have documented associations between decreased 25-hydroxyvitamin D (25D) levels and increased risk of adverse outcomes including prolonged length of stay [3], infection [4], acute kidney injury (AKI) [5], and mortality [3-7]. These associations are often attributed to the effects of vitamin D metabolites on host defense, which have been well characterized in vitro [8-10]. However, few studies in humans have simultaneously evaluated 25D levels together with markers of host defense in critically ill patients.

The strongest known link between vitamin D metabolites and the immune system relates to the effects of vitamin $\mathrm{D}$ metabolites on the production of cathelicidins

* Correspondence: deleaf@partners.org

Division of Renal Medicine, Brigham and Women's Hospital, 75 Francis Street, Boston, MA 02115, USA
[10], a critical family of antimicrobial proteins. Cathelicidins act by disrupting foreign-cell membranes, binding lipopolysaccharide residues, and recruiting leukocytes $[11,12]$. In animal models, deficiency of cathelicidin is associated with increased susceptibility to bacterial infection [13-15], whereas overexpression confers protection [16]. In humans, cathelicidin antimicrobial protein-18 (hCAP18) is the only known cathelicidin. hCAP18 is found primarily in the granules of neutrophils, and is also produced and secreted by monocytes, macrophages, epithelial cells, and other cell types [17]. Low 25D levels are associated with reduced production of hCAP18 by macrophages infected with Mycobacterium tuberculosis, whereas treatment with the activated vitamin D metabolite 1,25-dihydroxyvitamin $\mathrm{D}(1,25 \mathrm{D})$ in vitro results in enhanced production of hCAP18 and improved killing of the microorganisms [10]. In addition to macrophages, the in vitro inducibility of hCAP18 by $25 \mathrm{D}$ and $1,25 \mathrm{D}$ 
has also been demonstrated in multiple other human cell lines [10,18-22].

Despite the strong link between vitamin $\mathrm{D}$ metabolites and hCAP18 demonstrated in preclinical models, no study to our knowledge has measured plasma hCAP18 levels and evaluated their association with adverse outcomes in critically ill patients. Additionally, conflicting results have been reported on the association between plasma hCAP18 and 25D levels in humans [23-25]. We hypothesized that 25D and hCAP18 levels are directly correlated in critically ill patients, and that lower hCAP18 levels are associated with increased risk of 90-day mortality.

\section{Materials and methods Study design}

We conducted a prospective cohort study among patients admitted to intensive care units (ICUs) at Brigham and Women's Hospital between 2008 and 2012. Patients or their surrogates provided written informed consent and all protocols were approved by the Partners Human Research Committee (protocol \#2007P000894), which is the Institutional Review Board for Brigham and Women's Hospital.

Inclusion criteria were age $\geq 18$ years and admission to a medical or surgical ICU. Exclusion criteria were: (1) anticipated ICU stay <24 hours; (2) admitted to the ICU for a low-risk condition such as airway monitoring; (3) serum creatinine $>4.5 \mathrm{mg} / \mathrm{dl}$ or receiving dialysis; (4) pregnancy; and (5) institutionalized individuals.

We collected venous blood samples daily on ICU days 1 through 5. Blood samples were collected into EDTAcontaining vacutainers, centrifuged at 3,200 RPM for 15 minutes, and the plasma was aliquoted and stored at $-80^{\circ} \mathrm{C}$ within 2 hours of collection. We measured analytes in plasma samples at two time points: within 24 hours of ICU arrival and 48 hours later (hereafter referred to as ICU day 1 and 3, respectively).

\section{Clinical outcomes}

Investigator DEL adjudicated all outcomes by reviewing discharge summaries and progress notes, and was blinded to all study measurements at the time of adjudication. The primary endpoint was 90-day mortality. Secondary endpoints were hospital mortality, sepsis, incident AKI, duration of mechanical ventilation, and hospital length of stay. The association between hCAP18 levels and sepsis was assessed cross-sectionally since many of the patients already met sepsis criteria upon arrival to the ICU. Other outcomes were assessed prospectively.

Sepsis was defined according to consensus definition [26]. Incident AKI was defined according to serum creatinine-based criteria established by the Kidney Disease Improving Global Outcomes Work Group [27]. Patients who already had AKI $(\mathrm{N}=4)$ on arrival to the ICU were excluded from analyses of incident AKI. Duration of mechanical ventilation and hospital length of stay were assessed using ventilator-free days and hospital-free days to avoid the confounding effect of mortality. Ventilatorfree days and hospital-free days were defined as 28 minus the number of ventilator-dependent days or hospitalization days, respectively, assuming survival to 28 days or discharge from the hospital. Patients who died before 28 days were assigned a score of zero [28,29]. In exploratory analyses, we also assessed whether hCAP18 levels on ICU day 1 differed by sepsis severity, primary type of infection, or primary organism.

\section{Laboratory measurements}

All biomarkers were measured on ICU days 1 and 3 except parathyroid hormone (PTH), which was measured on ICU day 1 only. Assays were performed at the Harvard Medical School Clinical and Translational Science Award core laboratory. Plasma hCAP18 levels were measured by enzyme-linked immunosorbent assay (ELISA) using a commercially available kit (Hycult Biotech, Uden, Netherlands) which recognizes the 37 amino acid biologically active C-terminal fragment (LL-37) cleaved from hCAP18 [30]. Plasma 25D (combined $D_{2}$ and $D_{3}$, hereafter referred to as 'total 25D') and $D$ binding protein (DBP) levels were measured by ELISA using commercially available kits (Abbott Laboratories, Abbott Park, IL, USA and R\&D Systems, Inc., Minneapolis, MN, USA, respectively). Plasma intact PTH was measured using a chemiluminescent immunoassay (Beckman Coulter, Fullerton, CA, USA). Interassay coefficients of variation, estimated using blinded replicate samples from ICU patients, were $5.5 \%$ for hCAP18, 3.5\% for total 25D, $11 \%$ for DBP, and 3.1\% for PTH.

\section{Bioavailable and free 25D}

Because total 25D circulates bound to DBP (85 to 90\%) and albumin (10 to $15 \%$ ), less than $1 \%$ of circulating hormone exists in its free form [31]. The sum of free- and albumin-bound hormone is often referred to as 'bioavailable', because 25D bound to DBP is thought to have limited biological activity. In the absence of commercially available assays to directly measure bioavailable and free 25D levels, most studies have relied on formulas that incorporate binding coefficients for DBP and albumin, coupled to measurement of total 25D, DBP, and albumin levels [31-33]. Using these formulas, bioavailable compared to total 25D levels are more strongly associated with bone mineral density among young healthy adults [32]; serum calcium and PTH levels among incident hemodialysis patients [34]; and mortality among hospitalized patients [35]. We therefore used these equations to estimate bioavailable and free 25D levels. The 
Table 1 Baseline characteristics

\begin{tabular}{|c|c|}
\hline & $N=121$ \\
\hline \multicolumn{2}{|l|}{ Demographics } \\
\hline Age (yr) - median (IQR) & $62(56-73)$ \\
\hline Female sex (\%) & $46(38)$ \\
\hline White race (\%) & $112(93)$ \\
\hline \multicolumn{2}{|l|}{ Comorbidities (\%) } \\
\hline Hypertension & $64(53)$ \\
\hline Chronic lung disease & $37(31)$ \\
\hline Active malignancy & $34(28)$ \\
\hline Diabetes mellitus & $29(24)$ \\
\hline Chronic kidney disease & $7(6)$ \\
\hline Congestive heart failure & $4(3)$ \\
\hline Chronic liver disease & $2(2)$ \\
\hline \multicolumn{2}{|l|}{ Severity of illness } \\
\hline APACHE II score - median (IQR)* & $14(10-16)$ \\
\hline Charlson Comorbidity Index - median (IQR) ${ }^{\dagger}$ & $2(1-4)$ \\
\hline \multicolumn{2}{|l|}{ ICU type } \\
\hline Surgical & $102(84)$ \\
\hline SICU & $66(54)$ \\
\hline TICU & $36(30)$ \\
\hline Nonsurgical & $19(16)$ \\
\hline CCU & $11(9)$ \\
\hline $\mathrm{MICU}$ & $8(7)$ \\
\hline \multicolumn{2}{|l|}{ Status of procedure (for surgical patients) (\%) } \\
\hline Elective & $41(34)$ \\
\hline Urgent & $57(47)$ \\
\hline Days in hospital prior to enrollment - median (IQR) & $1(1-3)$ \\
\hline \multicolumn{2}{|c|}{$\begin{array}{l}\text { *APACHE II, Acute Physiology and Chronic Health Evaluation II, is an ICU } \\
\text { severity of illness scoring system ranging from } 0 \text { to } 71 \text {, with higher scores } \\
\text { corresponding to more severe disease; }{ }^{+} \text {Charlson Comorbidity Index is a } \\
\text { prognostic tool that has been validated in critically ill patients, with higher } \\
\text { scores indicating worse prognosis [37]. IQR, interquartile range; ICU, intensive } \\
\text { care unit; SICU, surgical/trauma ICU; TICU, thoracic ICU; CCU, cardiac care unit; } \\
\text { MICU, medical ICU. }\end{array}$} \\
\hline
\end{tabular}

equations used to calculate these levels are provided in Additional file 1.

\section{Statistical analyses}

Statistical analysis was performed with SAS Version 9.3 (SAS Institute Inc., Cary, NC, USA). Correlations between hCAP18 levels with total 25D, bioavailable 25D, free 25D, and PTH levels on ICU day 1 were assessed using Spearman's rank correlation coefficient. Comparison of biomarker levels between patients who were alive vs. dead at 90 days was assessed using the Wilcoxon rank sum test. Comparison of biomarker levels over time (ICU day 1 vs. day 3) was assessed using the Wilcoxon signed-rank test. Differences in hCAP18 levels on ICU day 1 on the basis of sepsis severity, primary type of infection, and primary organism were assessed using the Kruskal-Wallis test.

Logistic regression was used to assess the association between tertiles of biomarker levels on ICU day 1 and 90-day mortality (the primary outcome) as well as hospital mortality, sepsis, and AKI (secondary outcomes). The highest tertile served as the reference group. Multivariable models included age and acute physiology and chronic health evaluation (APACHE II) score, calculated on ICU day 1 . In exploratory analyses we also adjusted for ICU day 1 estimated glomerular filtration rate (eGFR), calculated using the chronic kidney disease epidemiology equation [36], since reduced kidney function could affect hCAP18 levels. Tertiles of biomarker levels on ICU day 1 and 90-day mortality were also assessed in aggregate using the Cochran-Armitage test for trend. The association between hCAP18 levels on ICU day 1 and ventilator-free days and hospital-free days was assessed using Spearman's rank correlation coefficient. All comparisons are two-tailed, with $P<0.05$ considered significant.

\section{Results}

We enrolled and collected samples from 121 patients. Median (interquartile range (IQR)) age was 62 (56 to 73) years. The most common comorbidities were hypertension

Table 2 hCAP18 and mineral metabolite/hormone levels on ICU days 1 and 3

\begin{tabular}{|c|c|c|c|c|}
\hline Biomarker & Reference range & ICU day $1(\mathrm{~N}=121)$ & ICU day $3(\mathrm{~N}=111)^{\dagger}$ & $P$ value \\
\hline hCAP18 (ng/ml) & Undefined & $160(106-286)$ & $166(116-291)$ & 0.92 \\
\hline Total 25D (ng/ml) & $30-80$ & $17(13-22)$ & $17(14-21)$ & 0.42 \\
\hline Bioavailable 25D (ng/ml) & Undefined & $2.5(1.8-3.5)$ & $2.4(1.6-3.3)$ & 0.24 \\
\hline Free 25D (pg/ml) & Undefined & $9.6(6.5-12.9)$ & $9.5(6.5-12.9)$ & 0.98 \\
\hline DBP (mg/dl) & 20-55 & $12.7(9.4-17.1)$ & $13.7(10.1-17.1)$ & 0.27 \\
\hline Albumin (g/dl) & $4.1-5.3$ & $3.1(2.4-3.5)$ & $2.9(2.3-3.3)$ & 0.002 \\
\hline PTH (pg/ml) & $15-65$ & $40(28-56)$ & --- & N/A \\
\hline
\end{tabular}

Values represent median ( $25^{\text {th }}$ to $75^{\text {th }}$ interquartile range). ${ }^{\dagger}$ Fewer samples were available on ICU day 3 due to interim death or discharge. ICU, intensive care unit; hCAP18, human cathelicidin antimicrobial protein-18; 25D, 25-hydroxyvitamin D; DBP, D-binding protein; PTH, parathyroid hormone. 

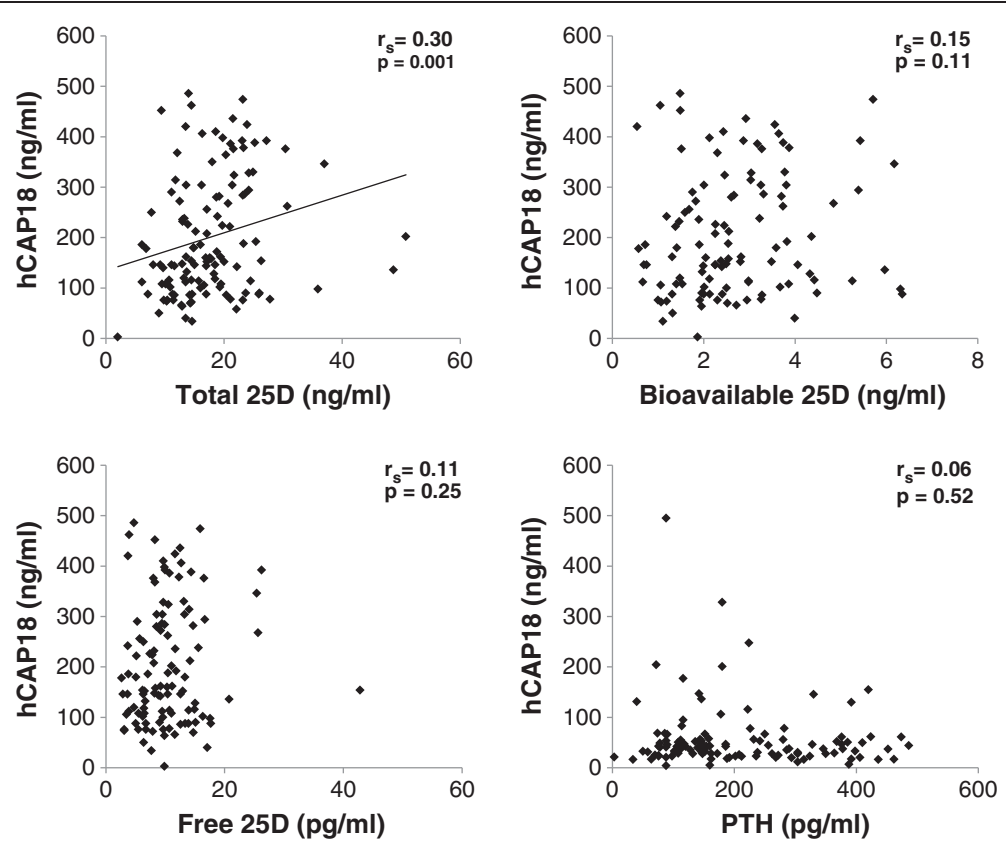

Figure 1 Correlations between plasma hCAP18, 25D metabolites, and PTH. Total 25D represents the sum of all 25-hydroxyvitamin D (both $D_{2}$ and $D_{3}$ ), including $25 D$ bound to DBP, albumin, and circulating freely; bioavailable 25D is the sum of albumin-bound and free 25D; free 25D is the circulating fraction of 25D that is neither bound to DBP nor albumin. DBP, D-binding protein; hCAP18, human cathelicidin antimicrobial protein-18; PTH, parathyroid hormone.

(53\%), chronic lung disease (31\%), and active malignancy (28\%). The majority of patients $(84 \%)$ were admitted to a surgical ICU. Additional baseline characteristics are shown in Table 1.

\section{hCAP18 and vitamin D markers}

Table 2 shows plasma levels of hCAP18, total 25D, bioavailable $25 \mathrm{D}$, and free $25 \mathrm{D}, \mathrm{DBP}$, albumin, and $\mathrm{PTH}$ from ICU days 1 and 3. Levels of total 25D on ICU day 1 were $<20 \mathrm{ng} / \mathrm{ml}$ in $68 \%$ of patients and $<30 \mathrm{ng} / \mathrm{ml}$ in $95 \%$ of patients. Other than albumin, which declined over time, plasma levels of other biomarkers were unchanged between ICU days 1 and 3 .

\section{Factors associated with hCAP18 levels}

Plasma hCAP18 levels on ICU day 1 correlated positively with total 25D (Spearman's rho $\left(\mathrm{r}_{\mathrm{s}}\right)=0.30, P=0.001$ ) but not with bioavailable $25 \mathrm{D}$, free $25 \mathrm{D}$, or $\mathrm{PTH}$ levels (Figure 1). Plasma hCAP18 levels on ICU day 1 did not differ by age, gender, type of ICU (surgical vs. nonsurgical), or status of procedure (urgent vs. elective). Similarly, plasma hCAP18 levels on ICU day 1 did not differ by comorbidities except for active malignancy (median (IQR) 120 (78 to 174) and 186 (116 to 304$) \mathrm{ng} / \mathrm{ml}$ for patients with vs. without active malignancy, $P=0.002$ ). Additionally, we found no correlation between hCAP18 levels and eGFR on ICU day 1 (Additional file 2).
Table 3 Sepsis characteristics

\begin{tabular}{|c|c|}
\hline & $\mathrm{N}=71$ \\
\hline \multicolumn{2}{|l|}{ Sepsis severity } \\
\hline Sepsis & $24(34)$ \\
\hline Severe sepsis & $30(42)$ \\
\hline Septic shock & $17(24)$ \\
\hline \multicolumn{2}{|l|}{ Primary site of infection } \\
\hline Respiratory & $35(49)$ \\
\hline Abscess or necrotizing fasciitis & $13(18)$ \\
\hline Gastrointestinal/peritonitis & $12(17)$ \\
\hline Unknown or other ${ }^{\dagger}$ & $11(16)$ \\
\hline \multicolumn{2}{|l|}{ Primary organism } \\
\hline Staphylococcus aureus & $14(20)$ \\
\hline Other $\operatorname{Gram}(+)^{\ddagger}$ & $13(18)$ \\
\hline Pseudomonas, Klebsiella, Escherichia coli, and Enterobacter & $15(21)$ \\
\hline Other Gram(-) & $8(11)$ \\
\hline Unknown or other ${ }^{\S}$ & $21(30)$ \\
\hline
\end{tabular}

Values represent $n(\%)$. Includes bacteremia $(\mathrm{N}=5)$, urosepsis $(\mathrm{N}=2)$, pericarditis $(\mathrm{N}=1)$, and septic arthritis $(\mathrm{N}=1)$; ${ }^{*}$ includes Enterococus $(\mathrm{N}=7)$, Lactobacillus $(\mathrm{N}=2)$, coagulase-negative Staphylococcus $(\mathrm{N}=2)$, Streptococcus pneumoniae $(\mathrm{N}=1)$, and Streptococcus mitis $(\mathrm{N}=1)$; ${ }^{\uparrow_{i n}}$ includes Proteus mirabilis $(N=2)$, Serratia $(N=2)$, Citrobacter $(N=1)$, Moraxella catarrhalis $(N=1)$, Fusobacterium necrophorum $(\mathrm{N}=1)$, and Hemophilus influenza $(\mathrm{N}=1)$; ${ }^{5}$ includes Clostridium difficile $(\mathrm{N}=3)$ and Aspergillus $(\mathrm{N}=1)$. 


\section{hCAP18 and adverse outcomes}

Among the 121 participants, 71 (59\%) had sepsis, 9 (7\%) died in the hospital, and 21 (17\%) died within 90 days. Data on sepsis severity, primary site of infection, and primary organism for the 71 patients who developed sepsis are shown in Table 3. Plasma hCAP18 levels on ICU day 1 did not differ by sepsis severity $(P=0.14)$, primary site of infection $(P=0.23)$, or primary organism $(P=0.07)$ (Additional file 3$)$.

Biomarker levels on ICU days 1 and 3 in patients who were alive vs. dead at 90 days are shown in Figure 2. Plasma hCAP18 levels on ICU day 1 were lower in patients who died compared to patients who survived (median (IQR) 116 (88 to 178) and 176 (112 to 304) $\mathrm{ng} / \mathrm{ml}, P=0.04)$. Additionally, free $25 \mathrm{D}$ levels on ICU day 3 were lower in patients who died compared to patients who survived (median (IQR) 7.7 (5.9 to 9.3) and 9.9 (7.0 to 13.1$), P=0.03$ ). Other biomarker levels were similar between survivors vs. nonsurvivors.

In univariate analyses, patients with ICU day 1 hCAP18 levels in the lowest vs. highest tertile had a significantly greater risk of 90-day mortality (Table 4). After adjusting for age and APACHE II score, the association was slightly attenuated but remained significant (adjusted odds ratio $=4.49,95 \%$ confidence interval $=$ 1.08 to $18.67, P=0.04$ ). In exploratory analyses, further adjustment for ICU day 1 eGFR did not affect the association (adjusted odds ratio $=4.44,95 \%$ confidence interval $=1.07$ to $18.45, P=0.04$ ). Tertiles of other biomarker levels on ICU day 1 were not associated with 90-day mortality (Table 4). We found similar findings when we analyzed the data using tests of trend across all three tertiles (Figure 3).

Table 5 shows the unadjusted and multivariableadjusted odds ratios evaluating the associations between ICU day 1 hCAP18 levels and hospital mortality, sepsis, and incident AKI. We found significant associations between hCAP18 levels in the lowest and middle tertiles and sepsis. We found no association between hCAP18 tertiles and hospital mortality or incident AKI.

Finally, we found no correlation between ICU day 1 hCAP18 levels and ventilator-free days $\left(r_{s}=-0.01\right.$, $P=0.96)$. We found a nonsignificant trend between hCAP18 levels and hospital-free days $\left(r_{\mathrm{s}}=0.16, P=0.06\right)$,
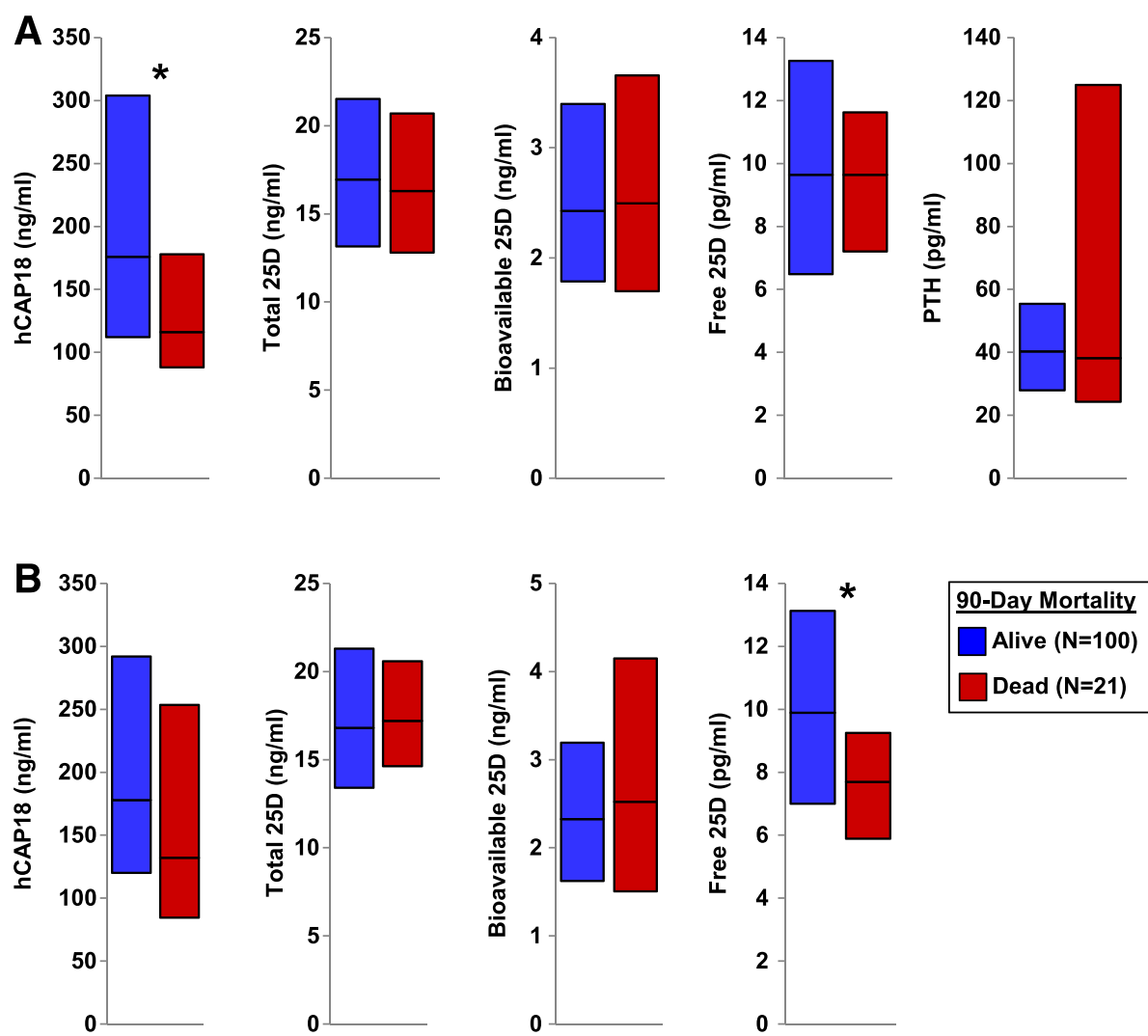

Figure 2 Plasma hCAP18, 25D metabolites, and PTH levels among survivors vs. nonsurvivors at 90 days. (A) Biomarker levels on ICU day 1. (B) Biomarker levels on ICU day $3 .{ }^{*} P<0.05$, comparison of biomarker levels among survivors vs. nonsurvivors. Bars represent median $\left(25^{\text {th }}\right.$ to $75^{\text {th }}$ interquartile range). 25D, 25-hydroxyvitamin D; hCAP18, human cathelicidin antimicrobial protein-18; ICU, intensive care unit; PTH, parathyroid hormone. 
Table 4 Unadjusted and multivariable-adjusted odds ratios comparing ICU day 1 levels of hCAP18, 25D metabolites, and PTH and risk of 90 -day mortality

\begin{tabular}{|c|c|c|c|c|}
\hline \multirow[t]{2}{*}{ Outcome } & \multicolumn{2}{|l|}{ Unadjusted } & \multicolumn{2}{|l|}{ Adjusted } \\
\hline & Odds ratio $(95 \% \mathrm{Cl})$ & $P$ value & Odds ratio $(95 \% \mathrm{Cl})$ & $P$ value \\
\hline \multicolumn{5}{|l|}{ hCAP18 } \\
\hline Tertile 1 & $4.80(1.23-18.80)$ & 0.02 & $4.49(1.08-18.67)$ & 0.04 \\
\hline Tertile 2 & $2.69(0.64-11.23)$ & 0.18 & $3.00(0.67-13.25)$ & 0.15 \\
\hline Tertile 3 (REF) & 1.00 & N/A & 1.00 & N/A \\
\hline \multicolumn{5}{|l|}{ Total 25D } \\
\hline Tertile 1 & $1.41(0.47-4.24)$ & 0.54 & $1.39(0.41-4.73)$ & 0.60 \\
\hline Tertile 2 & $0.69(0.21-2.40)$ & 0.56 & $0.69(0.19-2.56)$ & 0.58 \\
\hline Tertile 3 (REF) & 1.00 & N/A & 1.00 & N/A \\
\hline \multicolumn{5}{|l|}{ Bioavailable 25D } \\
\hline Tertile 1 & $0.97(0.28-3.34)$ & 0.96 & $1.11(0.28-4.36)$ & 0.88 \\
\hline Tertile 2 & $1.17(0.35-3.88)$ & 0.80 & $1.39(0.36-5.34)$ & 0.63 \\
\hline Tertile 3 (REF) & 1.00 & N/A & 1.00 & N/A \\
\hline \multicolumn{5}{|l|}{ Free 25D } \\
\hline Tertile 1 & $1.45(0.41-5.06)$ & 0.56 & $1.47(0.37-5.82)$ & 0.59 \\
\hline Tertile 2 & $1.45(0.41-5.06)$ & 0.56 & $1.88(0.44-8.05)$ & 0.40 \\
\hline Tertile 3 (REF) & 1.00 & N/A & 1.00 & N/A \\
\hline \multicolumn{5}{|l|}{ PTH } \\
\hline Tertile 1 & $1.00(0.31-3.19)$ & $>0.99$ & $1.22(0.36-4.13)$ & 0.75 \\
\hline Tertile 2 & $0.52(0.14-1.95)$ & 0.33 & $0.57(0.15-2.25)$ & 0.43 \\
\hline Tertile 3 (REF) & 1.00 & N/A & 1.00 & N/A \\
\hline
\end{tabular}

Twenty-one events. Adjusted models include age and APACHE II score. ICU, intensive care unit; hCAP18, human cathelicidin antimicrobial protein-18; 25D, 25-hydroxyvitamin D; PTH, parathyroid hormone; Cl, confidence interval; APACHE II, acute physiology and chronic health evaluation II.

implying a trend toward decreased hospital length of stay in patients with higher hCAP18 levels.

\section{Discussion}

In this prospective cohort study among critically ill patients, we found that lower total 25D levels were associated with lower hCAP18 levels, which were in turn associated with a greater risk of 90-day mortality. These findings provide a potential mechanistic basis for the commonly observed association between low 25D levels and poor outcomes in critically ill patients.

The current findings expand on prior studies of vitamin D metabolites and hCAP18. Preclinical studies have demonstrated potent antimicrobial effects of hCAP18 against a broad range of pathogens [13-16,38-43]. Further, the in vitro inducibility of hCAP18 by $25 \mathrm{D}$ and $1,25 \mathrm{D}$ has been demonstrated in multiple human cell lines [10,18-22]. These preclinical studies are complemented by numerous observational studies in humans, which have consistently shown associations between decreased 25D levels and increased mortality in patients with critical illness [3-7] and in other settings [44-46]. The association between decreased 25D levels and adverse outcomes in critical illness has been postulated to be mediated, at least in part, by decreased production of hCAP18 $[6,47,48]$, yet few studies have simultaneously measured 25D and hCAP18 and evaluated their association with adverse outcomes. None, to our knowledge, have done so in critically ill patients.

hCAP18 levels have been reported in critically ill patients in only two other studies, neither of which reported clinical outcomes. Jeng et al. found lower hCAP18 levels in critically ill patients $(\mathrm{N}=49)$ compared to healthy controls $(\mathrm{N}=21)$ [25]. In a larger study $(\mathrm{N}=130)$, no difference in hCAP18 levels was detected among ICU patients with vs. without sepsis [49]. A third study, which was conducted among hemodialysis outpatients, reported an inverse association between hCAP18 levels and 1-year infection-related mortality [24]. Consistent with these findings, we found associations between lower hCAP18 levels with both sepsis and 90-day mortality. Our findings raise the possibility that inadequate circulating levels of hCAP18, possibly due to decreased 25D levels, may predispose patients to infection and death. Consequently, randomized controlled trials (RCTs) are warranted to test whether administration of vitamin $\mathrm{D}$ metabolites may increase hCAP18 levels and thereby improve outcomes in critical illness. 


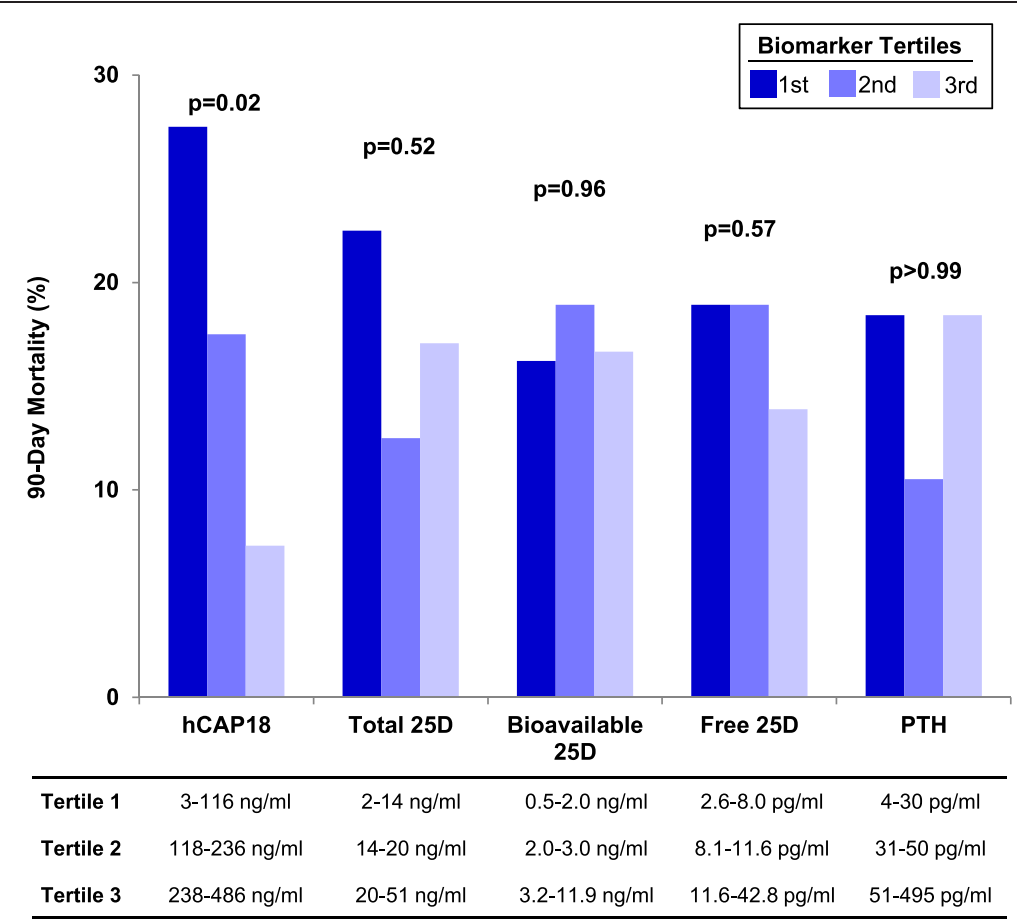

Figure 3 Tertiles of plasma hCAP18, 25D metabolites, and PTH on ICU day 1 and risk of 90-day mortality. 25D, 25-hydroxyvitamin D; hCAP18, human cathelicidin antimicrobial protein-18; ICU, intensive care unit; PTH, parathyroid hormone.

A recent RCT tested the effect of 540,000 IU of vitamin $\mathrm{D}_{3}$ in 492 critically ill patients [50]. Although the primary outcome of hospital length of stay was negative, a prespecified analysis of patients with severe vitamin $\mathrm{D}$ deficiency, defined as $25 \mathrm{D}$ levels $\leq 12 \mathrm{ng} / \mathrm{ml}$, found an almost $50 \%$ reduction in hospital mortality in the active treatment group compared to placebo. hCAP18 levels were not reported. In a pilot RCT of 67 critically ill patients with severe sepsis, we found that a single dose of calcitriol $(1,25 \mathrm{D})$ increased hCAP18 leukocyte mRNA expression at 24 hours but did not increase plasma levels [51]. Thus, additional RCTs are needed to evaluate whether hCAP18 is inducible in humans in response to vitamin D metabolites, as it

Table 5 Unadjusted and multivariable-adjusted odds ratios comparing ICU day 1 hCAP18 levels and secondary outcomes

\begin{tabular}{|c|c|c|c|c|}
\hline \multirow[t]{2}{*}{ Outcome } & \multicolumn{2}{|l|}{ Unadjusted } & \multicolumn{2}{|l|}{ Adjusted } \\
\hline & Odds ratio $(95 \% \mathrm{Cl})$ & $P$ value & Odds ratio $(95 \% \mathrm{Cl})$ & $P$ value \\
\hline \multicolumn{5}{|c|}{ Hospital mortality (9 events) } \\
\hline Tertile 1 & $2.17(0.37-12.55)$ & 0.39 & $1.97(0.33-11.75)$ & 0.46 \\
\hline Tertile 2 & $1.58(0.25-10.00)$ & 0.63 & $1.66(0.26-10.78)$ & 0.60 \\
\hline Tertile 3 (REF) & 1.00 & N/A & 1.00 & N/A \\
\hline \multicolumn{5}{|c|}{ Sepsis (71 events) } \\
\hline Tertile 1 & $2.35(0.96-5.74)$ & 0.06 & $2.54(1.01-6.4)$ & 0.047 \\
\hline Tertile 2 & $3.72(1.47-9.45)$ & 0.006 & $3.64(1.40-9.44)$ & 0.008 \\
\hline Tertile 3 (REF) & 1.00 & $\mathrm{~N} / \mathrm{A}$ & 1.00 & N/A \\
\hline \multicolumn{5}{|l|}{ AKI (33 events) } \\
\hline Tertile 1 & $0.65(0.22-1.96)$ & 0.45 & $0.61(0.20-1.88)$ & 0.39 \\
\hline Tertile 2 & $2.06(0.78-5.45)$ & 0.15 & $2.01(0.74-5.46)$ & 0.17 \\
\hline Tertile 3 (REF) & 1.00 & N/A & 1.00 & N/A \\
\hline
\end{tabular}

Adjusted models include age and APACHE II score. ICU, intensive care unit; hCAP18, human cathelicidin antimicrobial protein-18; Cl, confidence interval; AKI, acute kidney injury; APACHE II, Acute Physiology and Chronic Health Evaluation II. 
is in vitro, and whether upregulating hCAP18 improves outcomes.

We acknowledge the limitations of this study including single-center, a maximum of two data points per patient, and observational design. Although we measured DBP and $\mathrm{PTH}$, we did not measure all potentially relevant markers of mineral metabolism such as $1,25 \mathrm{D}$ and fibroblast growth factor-23, nor did we measure all potentially relevant markers of innate immunity, such as $\beta$ defensin 2 , which may also be upregulated by vitamin $\mathrm{D}$ metabolites [17]. Levels of bioavailable and free 25D were estimated using equations that were not developed and validated in critical illness. Therefore, estimated values for these vitamin D metabolites should be viewed as preliminary. Additionally, the association between hCAP18 levels on ICU day 1 and 90-day mortality could be confounded by a number of factors, such as severity of illness or comorbidities. Importantly, this association remained significant after adjusting for age and APACHE II score. Nonetheless, residual confounding from other variables cannot be excluded.

The association between hCAP18 levels and sepsis should be viewed cautiously for a variety of reasons. First, we did not have hCAP18 levels prior to the onset of acute illness and therefore cannot exclude the possibility of reverse causality, since sepsis was assessed cross-sectionally. Second, we cannot exclude the possibility of a type 1 error due to multiple comparisons. Finally, although approximately half of all sepsis cases were due to a respiratory infection, we did not find an association between hCAP18 levels and ventilator-free days, further suggesting that our finding of an association between hCAP18 levels and sepsis should be viewed as preliminary and in need of confirmation.

\section{Conclusions}

In conclusion, our central findings are that lower total 25D levels on ICU day 1 are associated with lower hCAP18 levels, which are in turn associated with a greater risk of 90-day mortality. These data support the notion that vitamin $\mathrm{D}$ metabolites may have important effects on innate immunity via induction of hCAP18. RCTs are needed to determine whether vitamin D metabolites upregulate hCAP18 in humans, as they do in vitro, and whether such a strategy will improve outcomes among critically ill patients.

\section{Key messages}

- hCAP18 levels on ICU day 1 correlate positively with total, but not bioavailable or free 25-hydroxyvitamin D levels.

- Lower hCAP18 levels on ICU day 1 are associated with increased risk of 90-day mortality.
- Vitamin D metabolites may have important effects on innate immunity via induction of hCAP18.

\section{Additional files}

Additional file 1: Supplemental methods. Calculation of bioavailable and free 25-hydroxyvitamin D levels.

Additional file 2: Plasma hCAP18 levels on ICU day 1 and eGFR. Plasma hCAP18 levels on ICU day 1 showed no correlation with eGFR.

Additional file 3: Plasma hCAP18 levels on ICU day 1 by (A) sepsis severity, (B) primary site of infection, and (C) primary type of organism.

\section{Abbreviations}

1,25D: 1,25-dihydroxyvitamin D; 25D: 25-hydroxyvitamin D; AKl: acute kidney injury; APACHE II: acute physiology and chronic health evaluation II; DBP: D-binding protein; eGRF: estimated glomerular filtration rate; ELISA: enzyme-linked immunosorbent assay; hCAP18: human cathelicidin antimicrobial protein-18; ICU: intensive care unit; IQR: interquartile range; PTH: parathyroid hormone; RCT: randomized controlled trial; $R_{s}$ : Spearman's rho.

\section{Competing interests}

The authors declare that they have no competing interests.

\section{Authors' contributions}

DEL and SSW designed the research. HEC, SJA, and AR recruited the patients, processed the samples, and assisted with chart review. DEL performed the statistical analyses and wrote the manuscript. All authors provided assistance in critically revising the manuscript, approved the final version to be published, and agreed to be accountable for all aspects of the work.

\section{Acknowledgements}

D.E.L. is supported by 1F32DK100040 from the National Institute of Diabetes and Digestive and Kidney Diseases. SSW is supported by R01DK093574 and U01DK085660. This work was conducted with support from Harvard Catalyst | The Harvard Clinical and Translational Science Center (National Center for Research Resources and the National Center for Advancing Translational Sciences, National Institutes of Health Award UL1 TR001102) and financial contributions from Harvard University and its affiliated academic healthcare centers. The content is solely the responsibility of the authors and does not necessarily represent the official views of Harvard Catalyst, Harvard University and its affiliated academic healthcare centers, or the National Institutes of Health.

Received: 31 October 2014 Accepted: 13 February 2015

Published online: 10 March 2015

\section{References}

1. Higgins DM, Wischmeyer PE, Queensland KM, Sillau SH, Sufit AJ, Heyland DK. Relationship of vitamin D deficiency to clinical outcomes in critically ill patients. JPEN J Parenter Enteral Nutr. 2012;36:713-20.

2. Lee P, Eisman JA, Center JR. Vitamin D deficiency in critically ill patients. N Engl J Med. 2009;360:1912-4

3. Matthews LR, Ahmed Y, Wilson KL, Griggs DD, Danner OK. Worsening severity of vitamin D deficiency is associated with increased length of stay, surgical intensive care unit cost, and mortality rate in surgical intensive care unit patients. Am J Surg. 2012;204:37-43.

4. Braun A, Chang D, Mahadevappa K, Gibbons FK, Liu Y, Giovannucci E, et al. Association of low serum 25-hydroxyvitamin D levels and mortality in the critically ill. Crit Care Med. 2011;39:671-7.

5. Braun $A B$, Litonjua AA, Moromizato T, Gibbons FK, Giovannucci E, Christopher KB. Association of low serum 25-hydroxyvitamin D levels and acute kidney injury in the critically ill. Crit Care Med. 2012;40:3170-9.

6. Arnson Y, Gringauz I, Itzhaky D, Amital H. Vitamin D deficiency is associated with poor outcomes and increased mortality in severely ill patients. QJM. 2012;105:633-9. 
7. Braun AB, Gibbons FK, Litonjua AA, Giovannucci E, Christopher KB. Low serum 25-hydroxyvitamin $D$ at critical care initiation is associated with increased mortality. Crit Care Med. 2012;40:63-72.

8. Mora JR, Iwata M, von Andrian UH. Vitamin effects on the immune system: vitamins A and D take centre stage. Nat Rev Immunol. 2008;8:685-98.

9. Hewison M. Antibacterial effects of vitamin D. Nat Rev Endocrinol. 2011;7:337-45

10. Liu PT, Stenger S, Li H, Wenzel L, Tan BH, Krutzik SR, et al. Toll-like receptor triggering of a vitamin D-mediated human antimicrobial response. Science. 2006:311:1770-3.

11. Zasloff M. Fighting infections with vitamin D. Nat Med. 2006;12:388-90

12. Turner J, Cho Y, Dinh NN, Waring AJ, Lehrer Rl. Activities of LL-37, a cathelin-associated antimicrobial peptide of human neutrophils. Antimicrob Agents Chemother. 1998;42:2206-14.

13. Radek KA, Elias PM, Taupenot L, Mahata SK, O'Connor DT, Gallo RL. Neuroendocrine nicotinic receptor activation increases susceptibility to bacterial infections by suppressing antimicrobial peptide production. Cell Host Microbe. 2010;7:277-89.

14. Huang LC, Reins RY, Gallo RL, McDermott AM. Cathelicidin-deficient (Cnlp -/- ) mice show increased susceptibility to Pseudomonas aeruginosa keratitis. Invest Ophthalmol Vis Sci. 2007:48:4498-508.

15. Nizet $V$, Ohtake T, Lauth $X$, Trowbridge J, Rudisill J, Dorschner RA, et al. Innate antimicrobial peptide protects the skin from invasive bacteria infection. Nature. 2001;414:454-7.

16. Bals R, Weiner DJ, Moscioni AD, Meegalla RL, Wilson JM. Augmentation of innate host defense by expression of a cathelicidin antimicrobial peptide. Infect Immun. 1999;67:6084-9.

17. Wang TT, Nestel FP, Bourdeau V, Nagai Y, Wang Q, Liao J, et al. Cutting edge: 1,25-dihydroxyvitamin D3 is a direct inducer of antimicrobial peptide gene expression. J Immunol. 2004;173:2909-12.

18. Dai X, Sayama K, Tohyama M, Shirakata Y, Hanakawa Y, Tokumaru S, et al. PPARgamma mediates innate immunity by regulating the 1alpha, 25-dihydroxyvitamin D3 induced hBD-3 and cathelicidin in human keratinocytes. J Dermatol Sci. 2010;60:179-86.

19. Yim S, Dhawan P, Ragunath C, Christakos S, Diamond G. Induction of cathelicidin in normal and CF bronchial epithelial cells by 1,25dihydroxyvitamin D(3). J Cyst Fibros. 2007;6:403-10.

20. McMahon L, Schwartz K, Yilmaz O, Brown E, Ryan LK, Diamond G. Vitamin D-mediated induction of innate immunity in gingival epithelial cells. Infect Immun. 2011;79:2250-6.

21. D'Aldebert E, Biyeyeme Bi Mve MJ, Mergey M, Wendum D, Firrincieli D, Coilly A, et al. Bile salts control the antimicrobial peptide cathelicidin through nuclear receptors in the human biliary epithelium. Gastroenterology. 2009;136:1435-43.

22. Adams JS, Ren S, Liu PT, Chun RF, Lagishetty V, Gombart AF, et al. Vitamin d-directed rheostatic regulation of monocyte antibacterial responses. J Immunol. 2009:182:4289-95.

23. Bhan I, Camargo Jr CA, Wenger J, Ricciardi C, Ye J, Borregaard N, et al. Circulating levels of 25-hydroxyvitamin D and human cathelicidin in healthy adults. J Allergy Clin Immunol. 2011; 127:1302-4. e1301.

24. Gombart AF, Bhan I, Borregaard N, Tamez H, Camargo Jr CA, Koeffler HP, et al. Low plasma level of cathelicidin antimicrobial peptide (hCAP18) predicts increased infectious disease mortality in patients undergoing hemodialysis. Clin Infect Dis. 2009;48:418-24.

25. Jeng L, Yamshchikov AV, Judd SE, Blumberg HM, Martin GS, Ziegler TR, et al. Alterations in vitamin D status and anti-microbial peptide levels in patients in the intensive care unit with sepsis. J Transl Med. 2009:7:28.

26. Levy MM, Fink MP, Marshall JC, Abraham E, Angus D, Cook D, et al. 2001 SCCM/ESICM/ACCP/ATS/SIS International Sepsis Definitions Conference. Crit Care Med. 2003;31:1250-6.

27. Kidney Disease; Improving Global Outcomes (KDIGO) Acute Kidney Injury Work Group. KDIGO clinical practice guideline for acute kidney injury. Kidney Int. 2012; Suppl 2:1-138.

28. Rubenfeld GD, Angus DC, Pinsky MR, Curtis JR, Connors Jr AF, Bernard GR. Outcomes research in critical care: results of the American Thoracic Society Critical Care Assembly Workshop on Outcomes Research: The Members of the Outcomes Research Workshop. Am J Respir Crit Care Med. 1999:160:358-67.

29. Contentin L, Ehrmann S, Giraudeau B. Heterogeneity in the definition of mechanical ventilation duration and ventilator-free days. Am J Respir Crit Care Med. 2014;189:998-1002.
30. Sorensen O, Cowland JB, Askaa J, Borregaard N. An ELISA for hCAP-18, the cathelicidin present in human neutrophils and plasma. J Immunol Methods. 1997;206:53-9.

31. Bikle DD, Gee E, Halloran B, Kowalski MA, Ryzen E, Haddad JG. Assessment of the free fraction of 25 -hydroxyvitamin $D$ in serum and its regulation by albumin and the vitamin D-binding protein. J Clin Endocrinol Metab. 1986;63:954-9.

32. Powe $C E$, Ricciardi C, Berg AH, Erdenesanaa D, Collerone G, Ankers E, et al. Vitamin D-binding protein modifies the vitamin D-bone mineral density relationship. J Bone Miner Res. 2011;26:1609-16.

33. Powe CE, Evans MK, Wenger J, Zonderman AB, Berg AH, Nalls M, et al. Vitamin D-binding protein and vitamin D status of black Americans and white Americans. N Engl J Med. 2013;369:1991-2000.

34. Bhan I, Powe CE, Berg AH, Ankers E, Wenger JB, Karumanchi SA, et al. Bioavailable vitamin $\mathrm{D}$ is more tightly linked to mineral metabolism than total vitamin D in incident hemodialysis patients. Kidney Int. 2012;82:84-9.

35. Leaf DE, Waikar SS, Wolf M, Cremers S, Bhan I, Stern L. Dysregulated mineral metabolism in patients with acute kidney injury and risk of adverse outcomes. Clin Endocrinol. 2013;79:491-8.

36. Levey AS, Stevens LA, Schmid CH, Zhang YL, Castro 3rd AF, Feldman HI, et al. A new equation to estimate glomerular filtration rate. Ann Intern Med. 2009;150:604-12

37. Charlson ME, Pompei P, Ales KL, MacKenzie CR. A new method of classifying prognostic comorbidity in longitudinal studies: development and validation. J Chronic Dis. 1987;40:373-83

38. Dorschner RA, Pestonjamasp VK, Tamakuwala S, Ohtake T, Rudisill J, Nizet V, et al. Cutaneous injury induces the release of cathelicidin anti-microbial peptides active against group A Streptococcus. J Invest Dermatol. 2001;117:91-7.

39. Howell MD, Jones JF, Kisich KO, Streib JE, Gallo RL, Leung DY. Selective killing of vaccinia virus by LL-37: implications for eczema vaccinatum. J Immunol. 2004;172:1763-7.

40. Barlow PG, Svoboda P, Mackellar A, Nash AA, York IA, Pohl J, et al. Antiviral activity and increased host defense against influenza infection elicited by the human cathelicidin LL-37. PLoS One. 2011;6:e25333.

41. Bergman P, Walter-Jallow L, Broliden K, Agerberth B, Soderlund J. The antimicrobial peptide LL-37 inhibits HIV-1 replication. Curr HIV Res. 2007:5:410-5.

42. Lai Y, Adhikarakunnathu S, Bhardwaj K, Ranjith-Kumar CT, Wen Y, Jordan JL, et al. LL37 and cationic peptides enhance TLR3 signaling by viral double-stranded RNAs. PLoS One. 2011:6:e26632.

43. Lopez-Garcia B, Lee PH, Yamasaki K, Gallo RL. Anti-fungal activity of cathelicidins and their potential role in Candida albicans skin infection. J Invest Dermatol. 2005;125:108-15.

44. Samefors M, Ostgren CJ, Molstad S, Lannering C, Midlov P, Tengblad A Vitamin D deficiency in elderly people in Swedish Nursing Homes is associated with increased mortality. Eur J Endocrinol. 2014;170:667-75.

45. Mehrotra R, Kermah DA, Salusky IB, Wolf MS, Thadhani RI, Chiu YW, et al. Chronic kidney disease, hypovitaminosis D, and mortality in the United States. Kidney Int. 2009;76:977-83.

46. Dobnig H, Pilz S, Scharnagl H, Renner W, Seelhorst U, Wellnitz B, et al. Independent association of low serum 25-hydroxyvitamin $d$ and 1,25-dihydroxyvitamin d levels with all-cause and cardiovascular mortality. Arch Intern Med. 2008;168:1340-9.

47. Izadpanah $\mathrm{M}$, Khalili $\mathrm{H}$. Potential benefits of vitamin D supplementation in critically ill patients. Immunotherapy. 2013;5:843-53.

48. van der Does AM, Bergman P, Agerberth B, Lindbom L. Induction of the human cathelicidin LL-37 as a novel treatment against bacterial infections. J Leukoc Biol. 2012:92:735-42.

49. Barbeiro DF, Barbeiro HV, Zampieri FG, Cesar Machado MC, Torggler Filho F, Gomes Cunha DM, et al. Cathelicidin LL-37 bloodstream surveillance is down regulated during septic shock. Microbes Infect. 2013;15:342-6.

50. Amrein K, Schnedl C, Holl A, Riedl R, Christopher KB, Pachler C, et al. Effect of high-dose vitamin D3 on hospital length of stay in critically III patients with vitamin D deficiency: the VITdAL-ICU randomized clinical trial. JAMA. 2014;312:1520-30.

51. Leaf DE, Raed A, Donnino MW, Ginde AA, Waikar SS. Randomized controlled trial of calcitriol in severe sepsis. Am J Respir Crit Care Med. 2014;190:533-41. 